\title{
Exponential peak and scaling of work fluctuations in modulated systems
}

\author{
M.I. Dykman \\ Department of Physics and Astronomy, Michigan State University, East Lansing, MI 48824, USA
}

(Dated: November 9, 2018)

\begin{abstract}
We extend the stationary-state work fluctuation theorem to periodically modulated nonlinear systems. Such systems often have coexisting stable periodic states. We show that work fluctuations sharply increase near a kinetic phase transition where the state populations are close to each other. The work variance is proportional here to the reciprocal rate of interstate switching. We also show that the variance displays scaling with the distance to a bifurcation point and find the critical exponent for a saddle-node bifurcation.

PACS numbers: 05.40.-a, 05.70.Ln, 74.50.+r, 85.85.+j
\end{abstract}

Since their discovery in the early 90 s 1 , 2, 3], fluctuation theorems have been attracting increasing interest. They establish general features of fluctuating systems away from thermal equilibrium, see Refs. 4,5 for reviews. A major "test bed" for fluctuation theorems is provided by dynamical systems with a few degrees of freedom coupled to a thermal bath, a Brownian particle being an example. Much of the corresponding theoretical and experimental work refers to (i) modulated linear systems, where fluctuations have been studied both in transient and stationary regimes [6, 7, 8, 9, 10, 11, 12], and (ii) nonlinear systems, initially at thermal equilibrium, driven to a different, generally nonequilibrium state [13, 14, 15, 16, 17].

Fluctuations in nonequilibrium dynamical systems have been attracting attention also in a different context. They play an important role in various types of mesoscopic vibrational systems of current interest. Because damping of the vibrations is typically weak, even a moderately strong resonant force can excite them to comparatively large amplitudes, where the nonlinearity becomes substantial. As a result, the system may have two or more coexisting stable states of forced vibrations [18]. Fluctuations can cause switching between these states [19] and thus significantly affect the overall behavior of the system even where they are small on average. Many features of the switching behavior and a range of phenomena and applications related to the switching, from quantum measurements to resonant frequency mixing and to high-frequency stochastic resonance have been studied experimentally [20, 21, 22, 23, 24, 25, 26, 27, 28].

In this paper we analyze work fluctuations in periodically modulated nonlinear dynamical systems coupled to a bath. We derive the stationary state work fluctuation theorem and show that, under fairly general assumptions, the distribution of fluctuations of work done by the modulating force over a long time $\tau$ is Gaussian. In common with systems close to thermal equilibrium, the work variance $\sigma^{2}$ is proportional to the average work $\langle W\rangle$, but the proportionality coefficient is not universal and depends on system parameters. It becomes exponentially large in bistable systems in the range of a kinetic phase transition where the stationary populations of the vibrational states are close to each other. This parameter range has similarity with the region of a first-order phase transition where molar fractions of the coexisting phases are close to each other [19, 29, 30].

Large work fluctuations are related to the difference in the power absorbed from the force in different vibrational states $i=1,2$. Switching back and forth between the states leads to significant fluctuations of the absorbed power. Their correlation time is determined by the switching (transition) rates. For a small characteristic intensity $D$ of the noise that comes from the bath, these rates are small compared to the dynamical relaxation rate in the absence of noise $t_{\mathrm{r}}^{-1}$ and the modulation frequency $\omega_{F}$. Then of primary interest are periodaveraged transition rates $\nu_{i j}$. They often display activation dependence on $D$, with $\nu_{i j} \propto \exp \left(-R_{i} / D\right)$, where $R_{i}$ is the characteristic activation energy of a transition $i \rightarrow j$. Since work fluctuations accumulate power fluctuations, and the typical accumulation time for interstate fluctuations is $\sim \nu_{i j}^{-1}$, the exponential smallness of $\nu_{i j}$ leads to an exponentially large factor in the variance $\sigma^{2}$.

We present the results for a most simple model: a nonlinear classical dynamical system modulated by a periodic force $F(t)=\sum_{n} \tilde{F}(n) \exp \left(i n \omega_{F} t\right)$; the coupling energy is $-F(t) q$, where $q$ is the system coordinate. The system is additionally coupled to a bath, which leads to relaxation and fluctuations. Work done by the force over time $\tau$ is

$$
W \equiv W(\tau)=\int_{0}^{\tau} d t F(t) \dot{q}(t)
$$

We are interested primarily in steady-state fluctuations, i.e., we assume that the system had come to the steady state well before the time $t=0$ when the work (11) started to be measured. This steady state is periodic in time with modulation period $\tau_{F}=2 \pi / \omega_{F}$. We further assume that correlations in the system decay sufficiently fast, for example, exponentially for long times, and that the time $\tau$ largely exceeds the characteristic correlation time of the system $t_{\text {corr }}$. Often for bistable systems $t_{\text {corr }} \sim 1 / \nu_{i j}$.

Work fluctuations can be expressed in terms of the correlation function of velocity fluctuations $Q\left(t, t^{\prime}\right)=$ $\left\langle\delta \dot{q}(t) \delta \dot{q}\left(t^{\prime}\right)\right\rangle$, where $\langle\ldots\rangle$ means ensemble average and $\delta \dot{q}(t)=\dot{q}(t)-\langle\dot{q}(t)\rangle$. Because the system is in a steady periodic state, we have $Q\left(t, t^{\prime}\right)=Q\left(t+\tau_{F}, t^{\prime}+\tau_{F}\right)$, and 
therefore

$$
Q\left(t, t^{\prime}\right)=\sum_{n} Q\left(n ; t-t^{\prime}\right) \exp \left[i n \omega_{F}\left(t+t^{\prime}\right) / 2\right] .
$$

We first consider the variance of the work distribution $\sigma^{2} \equiv \sigma^{2}(\tau)=\left\langle(\delta W)^{2}\right\rangle$, where $\delta W=W(\tau)-\langle W(\tau)\rangle$. In the limit of large $\tau$

$$
\begin{aligned}
& \sigma^{2} \approx 2 \pi \tau \sum_{n, m} \tilde{F}(n) \tilde{F}^{*}(m) \tilde{Q}\left(m-n ; \frac{n+m}{2} \omega_{F}\right), \\
& \tilde{Q}(n ; \omega)=(2 \pi)^{-1} \int_{-\infty}^{\infty} d t e^{i \omega t} Q_{n}(t) .
\end{aligned}
$$

Here we have taken into account that the correlation functions $Q\left(n ; t-t^{\prime}\right)$ decay on time $t_{\text {corr }}$ much smaller than $\tau$. Therefore the limits of integration over $t-t^{\prime}$ could be extended from $-\infty$ to $\infty$.

Decay of correlations on a time scale small compared to $\tau$ allows one to simplify the expressions for higher-order moments of $\delta W$ in a standard way. The 3rd moment $\left\langle(\delta W)^{3}\right\rangle$ is determined by an integral over $t_{1}, t_{2}, t_{3}$ of the correlator $\left\langle\delta \dot{q}\left(t_{1}\right) \delta \dot{q}\left(t_{2}\right) \delta \dot{q}\left(t_{3}\right)\right\rangle$. Because $\left|t_{1}-t_{2}\right|,\left|t_{1}-t_{3}\right| \lesssim t_{\text {corr }}$, we have $\left\langle(\delta W)^{3}\right\rangle \propto \tau$ for large $\tau$, and therefore $\left\langle(\delta W)^{3}\right\rangle /\left\langle(\delta W)^{2}\right\rangle^{3 / 2} \propto \tau^{-1 / 2}$, i.e., the $3 \mathrm{rd}$ moment is small for large $\tau$. The 4 th moment $\left\langle(\delta W)^{4}\right\rangle$ is determined by the integral of the correlator $\left\langle\delta \dot{q}\left(t_{1}\right) \delta \dot{q}\left(t_{2}\right) \delta \dot{q}\left(t_{3}\right) \delta \dot{q}\left(t_{4}\right)\right\rangle$. The main contribution to this integral comes from decoupling the correlator into pairs $\left\langle\delta \dot{q}\left(t_{n_{1}}\right) \delta \dot{q}\left(t_{n_{2}}\right)\right\rangle\left\langle\delta \dot{q}\left(t_{n_{3}}\right) \delta \dot{q}\left(t_{n_{4}}\right)\right\rangle$ with $\left|t_{n_{1}}-t_{n_{2}}\right|,\left|t_{n_{3}}-t_{n_{4}}\right| \lesssim t_{\text {corr }}$ while $\left|t_{n_{1}}-t_{n_{3}}\right| \sim \tau$ $\left(n_{i}=1, \ldots, 4\right)$. This gives $\left\langle(\delta W)^{4}\right\rangle \approx 3\left\langle(\delta W)^{2}\right\rangle^{2} \propto \tau^{2}$. Higher-order correlations in $\left\langle(\delta W)^{4}\right\rangle$ give a comparatively small contribution $\propto \tau$. The analysis can be immediately extended to higher moments of $\delta W$. It shows that the overall distribution of work fluctuations $P(W)$ is Gaussian,

$$
P(W)=\left(2 \pi \sigma^{2}\right)^{-1 / 2} \exp \left[-(W-\langle W\rangle)^{2} / 2 \sigma^{2}\right] .
$$

It follows from Eq. (3) that $P(W) / P(-W)=$ $\exp \left(2 W\langle W\rangle / \sigma^{2}\right)$, as in the stationary state fluctuation theorem for systems close to thermal equilibrium and for modulated linear systems, and the variance of the work distribution $\sigma^{2} \propto \tau \propto\langle W\rangle$. However, for strong periodic modulation there is no known general expression that would relate the average velocity $\langle\dot{q}(t)\rangle$ to the modulating force in terms of the correlation functions $\tilde{Q}_{n}(\omega)$. Moreover, as we show, the ratio $\sigma^{2} /\langle W\rangle$ may display sharp narrow peaks as a function of system parameters.

For weak noise, a system with two coexisting stable states $j=1,2$ mostly performs small fluctuations about these states and only occasionally switches between them. Then to leading order in the noise intensity $D$ the average work is a sum of partial works $W_{1,2}$ in each of the states weighted with the stationary populations of the states $w_{1,2}^{\mathrm{st}}[19]$,

$$
\begin{aligned}
& \langle W\rangle=\sum_{j=1,2} w_{j}^{\mathrm{st}} W_{j}, \quad W_{j}=\omega_{F} \tau \sum_{n} i n \tilde{F}^{*}(n) \tilde{q}_{j}(n), \\
& w_{1}^{\mathrm{st}}=\nu_{21} /\left(\nu_{12}+\nu_{21}\right), \quad w_{2}^{\mathrm{st}}=1-w_{1}^{\mathrm{st}} .
\end{aligned}
$$

Here, $\tilde{q}_{j}(n)$ is the Fourier component of the coordinate $q_{j}(t)$ in a periodic state $j, q_{j}(t)=\sum_{n} \tilde{q}_{j}(n) \exp \left(i n \omega_{F} t\right)$.

In contrast to the average work, the variance $\sigma^{2}$ has contributions of two different types. One comes from small-amplitude fluctuations about the stable states. It is given by the sum of partial variances $\sigma_{1,2}^{2}$ weighted with the state populations. The variances $\sigma_{1,2}^{2}$ can be obtained by linearizing equations of motion about the corresponding stable state and can be written as

$$
\sigma_{j}^{2}=C_{j} D W_{j}, \quad j=1,2 .
$$

The constant $C_{j}$ depends on the details of the system dynamics. For a system coupled to a thermal bath at temperature $T$, in the weak-field limit we have $\sigma_{j}^{2}=$ $2 k_{B} T W_{j}$. However, for strong field this relation does not hold in nonlinear systems, generally.

Fluctuations about $q_{j}$ become large near a saddle-node bifurcation point where the state $j$ disappears. Here, one of the motions of the system is slow [31], i.e., there is a "soft mode". Near the bifurcational (critical) position of the periodic state $q_{j}^{(c)}(t)$ one can quite generally write $q(t)-q_{j}^{(c)}(t)=q_{\mathrm{sm}}(t) \varkappa(t)$, where $\varkappa(t)=\varkappa\left(t+\tau_{F}\right)$ is a periodic function, whereas $q_{\mathrm{sm}}(t)$ is a slowly varying amplitude that depends on initial conditions. After rescaling the equation of motion for $q_{\mathrm{sm}}(t)$ can be written as [32]

$$
\left.\dot{q}_{\mathrm{sm}}=q_{\mathrm{sm}}^{2}-\eta+f(t), \quad\langle f(t) f) t^{\prime}\right)=2 D^{\prime} \delta\left(t-t^{\prime}\right) .
$$

Here, $\eta$ is the distance to the bifurcation point, for example, the scaled difference between the amplitude $A$ of the field and its critical value $A_{c}$ at the bifurcation point. The noise $f(t)$ is assumed to be white because of the slowness of $q_{\mathrm{sm}}(t)$; its intensity is $D^{\prime} \propto D$.

For $\eta>0$ in the absence of noise the system (7) has a stable state where $q_{\mathrm{sm}}=-\eta^{1 / 2}$. Small fluctuations about this state have variance $D^{\prime} \eta^{-1 / 2} / 2$ and decay over time $t_{\mathrm{r}}=\eta^{-1 / 2} / 2$. Therefore, from Eq. (3), near the bifurcation point where a state $j$ disappears

$$
\sigma_{j}^{2} / W_{j}=\tilde{C}_{j} D / \eta
$$

Factor $\tilde{C}_{j}$ is independent of $D$ and $\eta$, and $W_{j}$ does not diverge for $\eta \rightarrow 0$.

Equation (8) shows that the partial work variance scales as $\eta^{\xi}$ with the distance to the bifurcation point. The critical exponent is $\xi=-1$.

The other contribution to $\sigma^{2}$ comes from fluctuationinduced transitions between the states. The transitions lead to fluctuations of the state populations $w_{j}(t)$. These fluctuations are slow,

$$
\begin{aligned}
\left\langle\delta w_{1}(t) \delta w_{1}\left(t^{\prime}\right)\right\rangle & =w_{1}^{\mathrm{st}} w_{2}^{\mathrm{st}} \exp \left[-\nu_{\mathrm{tr}}\left|t-t^{\prime}\right|\right] \\
\nu_{\mathrm{tr}} & =\nu_{12}+\nu_{21}
\end{aligned}
$$

where $\delta w_{1}(t)=w_{1}(t)-w_{1}^{\text {st }}=-\delta w_{2}(t)$. In turn, they lead to slow fluctuations of the velocity $\dot{q}(t) \approx \sum_{j} \dot{q}_{j}(t) w_{j}(t)$ with decay time given by the reciprocal total transition rate $\nu_{\mathrm{tr}}^{-1} \gg t_{\mathrm{r}}, \tau_{F}$. 
From Eqs. (2), (3), (5), (9), the contribution to the work variance from interstate transitions is

$$
\sigma_{\mathrm{tr}}^{2} \approx \mathcal{M}\left(\nu_{\mathrm{tr}} \tau\right)^{-1}\left(W_{1}-W_{2}\right)^{2}, \quad \mathcal{M}=2 w_{1}^{\mathrm{st}} w_{2}^{\mathrm{st}},
$$

and the total variance is

$$
\sigma^{2}=\sum_{j} w_{j}^{\mathrm{st}} \sigma_{j}^{2}+\sigma_{\mathrm{tr}}^{2} .
$$

Equation (10) is the central result of the paper. It shows that the work variance is proportional to the squared difference of the partial works in the stable states and is inversely proportional to the transition rate $\nu_{\mathrm{tr}}$. The rate $\nu_{\text {tr }} \propto \exp \left(-\min _{i} R_{i} / D\right)$ is exponentially small for small noise intensity. Respectively, the variance (10) can be exponentially large compared to the variance due to small fluctuations about attractors (6).

Factor $\mathcal{M}$ in Eq. (10) sharply depends on the parameters of the system and the field $F(t)$. It is small,

$$
\mathcal{M} \propto \exp \left[-\left|R_{1}-R_{2}\right| / D\right],
$$

except for a narrow range of the kinetic phase transition where the transition activation energies are close to each other, $\left|R_{1}-R_{2}\right| \lesssim D$. At its maximum $\mathcal{M}=1 / 2$. Factor $\mathcal{M}$ determines also the intensity of super narrow peaks (of width $\nu_{\text {tr }} \ll t_{\mathrm{r}}^{-1}$ ) in the power spectra of modulated bistable systems and the spectra of absorption/amplification of an additional field [19, 33]. Its exponential dependence on the distance to the kinetic phase transition was seen in the simulations [34, 35] and experiment [25, 36].

The average work $\langle W\rangle$, Eq. (5), switches between the values $W_{1}$ and $W_{2}$ when the system goes through the kinetic phase transition. Therefore the ratio $\sigma^{2} /\langle W\rangle \propto$ $\mathcal{M} \nu_{\text {tr }}^{-1}$ displays a sharp peak at the transition.

The above results can be illustrated using as an example a resonantly driven underdamped Duffing oscillator, a model that describes a number of systems studied in recent experiments. In the absence of noise the oscillator dynamics is described by the equation

$$
\ddot{q}+\omega_{0}^{2} q+\gamma q^{3}+2 \Gamma \dot{q}=A \cos \omega_{F} t .
$$

We assume that the detuning of the field frequency from the oscillator eigenfrequency $\delta \omega=\omega_{F}-\omega_{0}$ and the friction coefficient $\Gamma$ are small: $|\delta \omega|, \Gamma \ll \omega_{0}$. Then the oscillator can display bistability of forced vibrations already for a comparatively small driving amplitude $A$, where the vibrations remain almost sinusoidal, $q_{j}(t) \approx a_{j} \cos \left(\omega_{F} t+\phi_{j}\right)(j=1,2)$. Explicit expressions for the amplitudes $a_{1,2}$ and phases $\phi_{1,2}$ are well known [18]. The interstate transition rates are well understood also $19,21,22,24,35$.

The partial work in a stable vibrational state $j$ is $W_{j}=$ $\Gamma \tau \omega_{F}^{2} a_{j}^{2}$. By calculating the partial variances $\sigma_{j}^{2}$ due to thermal noise one obtains

$$
\begin{gathered}
\frac{\sigma_{j}^{2}}{W_{j}}=2 k_{B} T Z_{j}^{-2}\left[\left(Z_{j}-2\right)^{2}+4 \Omega^{2}\left(Y_{j}-1\right)^{2}\right], \\
\Omega=\delta \omega / \Gamma, \quad Z_{j}=1+\Omega^{2}\left(Y_{j}-1\right)\left(3 Y_{j}-1\right),
\end{gathered}
$$

FIG. 1: (Color online) Scaled ratios of the partial work variance to mean partial work $S_{j}=\sigma_{j}^{2} / 2 k_{B} T W_{j}, j=1,2$, as functions of the reduced squared modulation amplitude $\beta$ for a Duffing oscillator; $\Omega^{-1}=\Gamma / \delta \omega=0.3$. The curves 1 and 2 refer to large- and small-amplitude vibrations, respectively. The functions $S_{1,2}$ diverge at the corresponding bifurcation points of the oscillator, $\beta_{B}^{(1)} \approx 0.088$ and $\beta_{B}^{(2)} \approx 0.18$.

It should be possible to see the scaling (15) for modulated oscillators for the same conditions as the scaling of the switching activation energies [21, 24]. Similarly, the exponential peak of the work variance should be seen in experiments analogous to those where there were observed other kinetic phase transition phenomena like super narrow peaks in the power spectra, high-frequency stochastic resonance, and fluctuationenhanced frequency mixing [25, 28, 36]. 
Experiments on the scaling and on the kinetic phase transition should be conducted in a different way. The scaling can be observed in the quasistationary regime. The system should be prepared in the metastable state near a bifurcation point, and the duration of the experiment should be shorter than the lifetime of the state. In contrast, the exponential peak of the variance can be seen provided the duration of the measurement exceeds the reciprocal switching rate $\nu_{\mathrm{tr}}^{-1}$.

In conclusion, we have considered the stationary-state work fluctuation theorem for dynamical systems modulated by a strong periodic field. We have shown that, if the system has coexisting states of forced vibrations, work fluctuations may become strong. The ratio of their variance to average work is proportional to the reciprocal rate of interstate transitions. It displays a sharp exponentially high peak as a function of the distance to the kinetic phase transition. In a different parameter range, near a saddle-node bifurcation point where one of the stable vibrational states disappears, in the quasistationary regime the variance displays scaling with the distance to the bifurcation point.

I am grateful to E. G. D. Cohen for the insightful and stimulating discussion of the fluctuation theorems. This work was supported in part by the NSF through grant PHY-0555346.
[1] D. J. Evans, E. G. D. Cohen, and G. P. Morriss, Phys. Rev. Lett. 71, 2401 (1993).

[2] D. J. Evans and D. J. Searles, Phys. Rev. E 50, 1645 (1994).

[3] G. Gallavotti and E. G. D. Cohen, Phys. Rev. Lett. 74, 2694 (1995).

[4] D. J. Evans and D. J. Searles, Adv. Phys. 51, 1529 (2002).

[5] C. Bustamante, J. Liphardt, and F. Ritort, Phys. Today 58 (7), 43 (2005).

[6] G. M. Wang, E. M. Sevick, E. Mittag, D. J. Searles, and D. J. Evans, Phys. Rev. Lett. 89, 050601 (2002).

[7] R. van Zon, S. Ciliberto, and E. G. D. Cohen, Phys. Rev. Lett. 92, 130601 (2004).

[8] N. Garnier and S. Ciliberto, Phys. Rev. E 71, 060101 (2005).

[9] F. Zamponi, F. Bonetto, L. F. Cugliandolo, and J. Kurchan, J. Stat. Mech. p. P09013 (2005).

[10] E. G. D. Cohen and R. van Zon, cond-mat/0701683 (2007).

[11] S. Joubaud, N. Garnier, and S. Ciliberto, condmat/0703798 (2007).

[12] A. Imparato, L. Peliti, G. Pesce, G. Rusciano, and A. Sasso, cond-mat/0707.0439 (2007).

[13] C. Jarzynski, Phys. Rev. Lett. 78, 2690 (1997).

[14] G. E. Crooks, Phys. Rev. E 60, 2721 (1999).

[15] G. Hummer and A. Szabo, Proc. Nat. Acad. 98, 3658 (2001).

[16] D. Collin, F. Ritort, C. Jarzynski, S. B. Smith, I. Tinoco, and C. Bustamante, Nature 437, 231 (2005).

[17] V. Blickle, T. Speck, L. Helden, U. Seifert, and C. Bechinger, Phys. Rev. Lett. 96, 070603 (2006).

[18] L. D. Landau and E. M. Lifshitz, Mechanics (Elsevier, Amsterdam, 2004), 3rd ed.

[19] M. I. Dykman and M. A. Krivoglaz, Zh. Eksp. Teor. Fiz. 77, 60 (1979).

[20] L. J. Lapidus, D. Enzer, and G. Gabrielse, Phys. Rev. Lett. 83, 899 (1999).
[21] I. Siddiqi, R. Vijay, F. Pierre, C. M. Wilson, L. Frunzio, M. Metcalfe, C. Rigetti, and M. H. Devoret, condmat/0507248 (2005).

[22] J. S. Aldridge and A. N. Cleland, Phys. Rev. Lett. 94, 156403 (2005).

[23] K. Kim, M. S. Heo, K. H. Lee, H. J. Ha, K. Jang, H. R. Noh, and W. Jhe, Phys. Rev. A 72, 053402 (2005).

[24] C. Stambaugh and H. B. Chan, Phys. Rev. B 73, 172302 (2006).

[25] C. Stambaugh and H. B. Chan, Phys. Rev. Lett. 97, 110602 (2006).

[26] I. Siddiqi, R. Vijay, M. Metcalfe, E. Boaknin, L. Frunzio, R. J. Schoelkopf, and M. H. Devoret, Phys. Rev. B 73, 054510 (2006).

[27] B. Abdo, E. Segev, O. Shtempluck, and E. Buks, J. Appl. Phys. 101, 083909 (2007).

[28] R. Almog, S. Zaitsev, O. Shtempluck, and E. Buks, Appl. Phys. Lett. 90, 013508 (2007).

[29] R. Bonifacio and L. A. Lugiato, Phys. Rev. Lett. 40, 1023 (1978).

[30] L. A. Lugiato, Prog. Opt. 21, 69 (1984).

[31] J. Guckenheimer and P. Holmes, Nonlinear Oscillators, Dynamical Systems and Bifurcations of Vector Fields (Springer-Verlag, New York, 1987).

[32] M. I. Dykman, B. Golding, and D. Ryvkine, Phys. Rev. Lett. 92, 080602 (2004).

[33] M. I. Dykman, M. A. Krivoglaz, and S. M. Soskin, in Noise in Nonlinear Dynamical Systems, edited by P. V. E. McClintock and F. Moss (Cambridge University Press, Cambridge, 1989), vol. 2, pp. 347-380.

[34] M. I. Dykman, R. Mannella, P. V. E. McClintock, and N. G. Stocks, Phys. Rev. Lett. 65, 48 (1990).

[35] M. I. Dykman, D. G. Luchinsky, R. Mannella, P. V. E. McClintock, N. D. Stein, and N. G. Stocks, Phys. Rev. E 49, 1198 (1994).

[36] H. B. Chan and C. Stambaugh, Phys. Rev. B 73, 224301 (2006). 